\title{
Dressed up problems-the danger of picking the inappropriate dress
}

\author{
Anna Muzsnay and Csaba Szabó
}

\begin{abstract}
Modelling and dressed-up problems play an inevitably unavoidable role in mathematics education. In this study we would like to point out how dangerous is it to dress up mathematical problems. We go back to the principle of De Lange: The problem designer is not only dressing up the problem, but he is the solution designer, as well. We show three examples selected from Hungarian high school textbooks where the intended solution does not solve the problem, because the dressing changes the context and changes the problem itself.
\end{abstract}

Key words and phrases: dressed-up problems, modelling problems, problem creator.

ZDM Subject Classification: M10 D80 D30.

\section{Introduction}

The aim of the paper is to show how dangerous can be to dress up mathematical problems. We approach the "dresscode" from the point of view of the problem designer. The problem designer is not only dressing up the problem, but he is the solution designer, as well [13].

A possible classification splits math problems into three classes: dressedup word problems are one of the three types besides modelling problems and intra-mathematical problems [2]. We can distinguish them by their strength of connection to reality. Dressed up problems in this classification include simple 
word problems as well as context problems. We call a word problem a dressedup problem if for a purely mathematical problem a real life imitating context is created to make it a word problem.

By solving modelling problems we create connection between real and mathematical world. In [21] an idealized process of solution for a modelling problem is presented:

(1) understanding the problem and constructing an individual "situation model"

(2) simplifying and structuring the situation model and thus constructing a "real model"

(3) mathematizing, i.e. translating the real model into a mathematical model

(4) applying mathematical procedures in order to derive a result

(5) interpreting this mathematical result with regard to reality and thus attaining a real result

(6) validating this result with reference to the original situation and if the result is unsatisfactory, the process may start again with step 2

(7) exposing the whole solution process.

To simplify the process: we have a problem from the real world which we transfer to a mathematical problem (formulating), then we get a mathematical solution which we transfer to real world solution (interpretation) and validate the result. Intra-mathematical problems are mathematical problems without any connection to reality. An intra-mathematical problem can be solved using only mathematical procedures. Contrary to intra-mathematical problems "dressedup" word problems are related to reality, but not as strongly as modelling problems. The main differences between the two types:

- In a word problem the real model is already given in the task.

- The data for finding the solution are given in the text and no other data are needed for development of the solution.

- "Modelling loops" for validation of the real result are unnecessary.

Context problems are categorized by their direct or indirect relationship to reality (e.g. open or closed problems), and by the order of activities involved in the solution (open-open and closed-open questions) [13], as well.

We believe no one is shocked by the fact that in recent years more and more criticized contexts [18] of word problems. It is enough to look into a textbook and we can understand why a significant part of children are not that amazed by doing tasks from it. To show one we present Palm's instructive example. 
EXAMPLE 0.1. In a bakery you see a $20 \mathrm{~cm}$ long cylinder-shaped Swiss roll. A dissection straight through the cake produces a circular shape with a diameter of $7 \mathrm{~cm}$. The points of time in a day when the Swiss rolls are all sold are normally distributed with mean 5.30p.m. and standard deviation 15 minutes.

(1) What is the volume of the Swiss roll?

(2) What is the probability that the Swiss rolls are all sold before 6.00p.m., when the bakery closes?

There are several problems with this word-problem which are indicated in the the paper. One might ask the question: "Who cares the volume of the Swiss rolls?". After hearing this problem how many students ask the following questions from himself or from the teacher (even the motivated ones): "Why are we doing this?" "When would we ever need to know this in our lives?". Similar questions on dressed up problems are put in [22], saying that keeping relevancy, in the most honest sense of the word, at the heart of real-world problems must be our goal. One can notice that a really little part of these textbook tasks are real simulations of out-of-school situations. Boaler [3] shows that although real life situations (like shopping in word problems) do not necessarily aid in real life, but the complexity of issues relating to context, transfer and student's learning does not allow to dismiss the usefulness of context. Many researchers (and not only researchers) concern about this issue involving the sex of the student, [4, 5], cognitivity vs. stereotypy [10], or the review [20] on the weight and effect on context and understanding of the context in the solvability of a problem. For more theoretical reviews consult [16, 23]. Usually these problems are only 'dressed up' with an out-of-school figurative context, since out-of-school elements in mathematics education is obligated by policy documents in a wild range of countries. According to Gerofsky word problems can be seen as a genre "written in imitation not of life but of other word problems" [8].

One can ask the question if there are so many problems with these contexts than why we use it in mathematics education. Contexts have several benefits. According to Van Den Heuvel-Panhuizen [11] the main role of contexts are:

- enhancing the accessibility to problems

- contributing to the transparency and elasticity of problems

- suggesting solution strategies to students.

Problem-solving skills are of central importance in school mathematics achievement. A possible way to equip students with problem solving skills can 
be provided by Realistic Mathematics Education. Reality is a source for learning mathematics and as a consequence contexts are also useful in order to improve the ability of abstraction and developing mathematical tools. Goldin and Kaput [9] pointed out that the ability to visualize data in the sense of [19] in a mathematical problem may contribute to mathematics problem-solving. According to Lave [14], the importance of contexts lies not in the mathematical structure they reflect but in activities of the school system. Although contexts have several benefits, we cannot rule out that they require a certain level of reading skills. For example, in Hungary the roles and the importance of word problems are specified in the Hungarian National Core Curriculum (Nemzeti alaptanterv, 2007) [15] in that "the individual is able to apply basic mathematical principles and processes in acquiring knowledge and in solving problems in daily life, at home and at the workplace" (p. 9). As one can read in [7]: objectives of word problem solving are declared for school grades from 1 to 8, without detailing the steps or levels and without attaching them to grades or age groups. (one can find further information in the local curricula). But, as it is stated, no word problems can have an effect for pupils below grade 3 , before the basic reading skills are about starting to develop.

There are arguments and counterarguments concerning issues about wordproblems or context-problems. Besides modelling problems we need dressed-up word problems, as well. In this study we would like to point out how dangerous is it to dress up mathematical problems. We go back to the principle of De Lange [13]: The problem designer is not only dressing up the problem, but he is the solution designer, as well. We show three examples where the intended solution does not solve the problem, because the dressing changes the context and changes the problem itself. This happens especially when we want to give the context a modelling flavour. We demonstrate these hazards with a problem from analysis, combinatorics and geometry selected from Hungarian high school textbooks.

Note that although we are pointing at risks and strictly speaking mathematical mistakes, these are far from being typical. The goal of this paper is not to blame the authors. Every Hungarian textbook series is approved by the Ministry of Education or one of its organisations and every textbook has several (co)authors. These authors are experienced highschool teachers. We do not know and would not like to investigate the way these books were written. In particular, we do not claim the any single author is responsible for these problems. Thus in case of two of the three problems we show a few correctly handled problems, as well. These problems are posed for Hungarian highschool students grade 9-12 
of age tipically 14-19. We picked problems from grade 9 and grade 11 . For the reader to be a little bit familiar with the curricula, we list the titles of the chapters of textbooks at the corresponding subsections.

\section{Correct and incorrect solutions to word problems}

\section{Exponential growth}

The most well-known textbook [6] for grade 11 students involves 6 chapters

(1) Combinatorics, graphs

(2) Power, root, logarithm

(3) Applications of trigonometry

(4) Functions

(5) Analytic geometry

(6) Probability theory and statistics

Each chapter contains several sections and each section is closed with solved examples and/or exercises.

The chapter on logarithm is started with the following question with solution:

Problem 1. A copier costs 220000 HUF. During the use, the current value of the machine is reduced by 20 percent. When the machine is less than 50000 $H U F$, it is no longer economical to fix it in case of a bigger mistake. How many years later can it be said that if the copier is broken, it is worth replacing?

Solution 1. Under the condition the value of the machine: after 1 year

$$
220000 \cdot 0.8 H U F
$$

after 2 years

$$
(220000 \cdot 0.8) \cdot 0.8 H U F=220000 \cdot 0.8^{2} H U F
$$

after $\mathrm{x}$ years

$$
220000 \cdot 0.8^{x} H U F
$$


We want to find $x$, such that:

$$
\begin{gathered}
220000 \cdot 0.8^{x}=50000 \\
0.8^{x}=0.2273
\end{gathered}
$$

Take the decadic logarithm of both sides

$$
\begin{gathered}
\lg 0.8^{x}=\lg 0.2273 \\
x \cdot \lg 0.8=\lg 0.2273 \\
x=\frac{\lg 0.2273}{\lg 0.8}
\end{gathered}
$$

Using calculator we get

$$
x \approx 6.64
$$

After about 6.64 years, the cost of the copier is reduced to 50000 HUF, then it is no longer worth it to to fix the machine in case of a bigger mistake.

Another example from this chapter. After a short review of the behaviour of radioactivity and presenting the half-life of $\mathrm{C}$-14 isotope the following problem is posed.

Problem 2. According to the examination 4 of 12 gram carbon is radioactive in a finding at an excavation. Estimate the age of the finding.

Solution 2. In the knowledge of the half-life of C-14 isotope we can calculate the parameter $k$ for $\mathrm{C}-14$ :

$$
\begin{gathered}
\frac{m_{0}}{2}=m_{0} \cdot e^{-5715 \cdot k} \\
\frac{1}{2}=e^{-5715 \cdot k}
\end{gathered}
$$

Taking the natural logarithm of both sides

$$
\begin{aligned}
& \ln \frac{1}{2}=\ln e^{-5715 \cdot k} \\
& \ln \frac{1}{2}=-5715 \cdot k
\end{aligned}
$$


Using a calculator:

$$
k=\frac{\ln \frac{1}{2}}{-5715}=0.000121\left(\frac{1}{\text { year }}\right)
$$

Knowing that in the case of C-14

$$
m(t)=m_{0} \cdot e^{-0.000121 \cdot t}
$$

According to the measurement results

$$
\begin{gathered}
4=12 \cdot e^{-0.000121 \cdot t} \\
\frac{1}{3}=e^{-0.000121 \cdot t}
\end{gathered}
$$

Take the natural logarithm of both sides

$$
\begin{gathered}
\ln \frac{1}{3}=\ln e^{-0.000121 \cdot t} \\
\ln \frac{1}{3}=-0.000121 \cdot t \\
t=\frac{\ln \frac{1}{3}}{-0.000121} \approx 9079.4
\end{gathered}
$$

It means, that the finding is about 9079 years old.

The chapter on logarithm is closed with the following question with solution:

Problem 3. The table contains data from three different years on the number of deaths caused by drugs in Hungary. Give a prediction for the year 2010.

\begin{tabular}{|c|c|c|c|}
\hline year & 1995 & 1996 & 1997 \\
\hline number & 204 & 288 & 339 \\
\hline \multicolumn{4}{|c|}{ Table 1 }
\end{tabular}

Solution 3. This is a rapidly increasing process, so we can approximate with a $h(t)=h_{0} e^{k t}$ function. Let $h_{0}$ be the initial value from $1995, h_{0}=204$. We measure the elapsed time in years. We want to find $k$, the parameter of the process. 
I By the data from 1995. and 1996. we obtain

$$
\begin{aligned}
& 288=204 \cdot e^{k \cdot 1} \\
& \frac{288}{204}=e^{k}
\end{aligned}
$$

using calculator we get

$$
k=\ln \frac{288}{204} \approx 0.3448\left(\frac{1}{\text { year }}\right)
$$

II By the data from 1995. and 1997. we obtain

$$
\begin{aligned}
& 339=204 \cdot e^{k \cdot 2} \\
& \frac{339}{204}=e^{2 k}
\end{aligned}
$$

using calculator we get

$$
2 k=\ln \frac{339}{204} \approx 0.3448\left(\frac{1}{\text { year }}\right)
$$

Using calculator

$$
k=\frac{1}{2} \ln \frac{339}{204} \approx 0,2539\left(\frac{1}{\text { year }}\right)
$$

Then the book explains that the difference between the two values shows the need for more data for a more precise prediction. Then some conclusion is given that with more data we can give a better description of this phenomena originating from real life.

The first question arising is why this is a dressed up problem. How can one observe that the problem is not a modelling problem? The number of deaths caused by drugs in Hungary is between 15 and 30 from 2000-2016 according to both the national [25] both the European Union [26]. As one can see the number of deaths in Hungary is not close to 300, not growing, and in particular does not grow exponentially. What can be the reason that this problem in this form with this solution was put into the textbook. The first question is how a student could have known or predict that this is an exponential process. Especially, because it is not. In such problems either you give much more data, or present a parametric growth function, or you know from earlier experience that these real life processes have exponential growth. Clearly, a high school student of age 17-18 does not bear 
with any of these knowledge. If no parametric growth function is given then there is no chance to make predictions from three data. On three points almost any arbitrary curve can be interpolated. This can be a polynomial of degree at least 2 (Lagrange-interpolation), exponential, logarithmic or a saturation curve, etc. If you carefully examine the three numbers, $288-204>339-288$. Hence, as the process is not going fast, there is no reason to predict an exponentially growing procedure.

We wanted to explore where the dressing up procedure went wrong. Where the idea could originate from. In order to analyze the problem we interviewed three scholars if they have something in their mind about the background of this problem. We made interviews with Dr habil Tamás Pfeil, associate professor of applied analysis, an expert of differential equations and modelling, Dr Csaba Kerepesi, a brain researcher, having a $\mathrm{PhD}$ in informatics, a data miner expert and Gy. Szabó a student of biology.

Reading the task Tamás Pfeil said that this task is nonsense, because we can not give a prediction from only three samples. We need much more to estimate the process. We also asked him what could the author have thinking about. After a bit of hesitation Tamás Pfeil replied the followings: most likely the author picked out the first few data of a larger database. In these situations the phenomenons may seem exponential (despite of the function which describes the phenomenon is not exponential). After a careful examination of the textbook's solution, he had additional comments. He remarked that obviously the book's solution is incorrect. The reasoning of the discrepancy $k$ is not valid. The discrepancy shows that $h(t)$ is not an exponential function. In order to know more about the approximating function and to obtain a better approximation we need much more data. Furthermore, an attentive reader can remark that the mortality rates are decreasing, therefore the approximating function cannot have the form considered in the solution.

Dr. Csaba Kerepesi said that no process can be estimated from three data, not any conclusion can be drawn from three numbers, except if the growth function is given. We asked him about the possible origin of the problem. At first he had no idea, but he said the following: the instantaneous mortality rate is the expected rate at which people die per unit of time, in our case per year at each precise age, relative to the surviving population at that age. This means the following: The human cells at late ages are dying fast. And after the age of 70 they do it so fast, that the risk of death has really exponential growth from day to day. This mortality curve is exponential. But not the mortality curve of the 
problem. So the authors might have heard somewhere that: "The mortality curve is exponential" and they applied this sentence to the phenomena of deaths caused by drugs. Gyula Szabó first asked when the syringe change program took place. It is an important parameter to answer the question. He argued as Dr. Tamás Pfeil. Then he explained that in biology the corresponding curve is the so called survival curve. It has three types measuring the number of species surviving in a biologically closed population. None of the three types is exponential.

All three scholars gave enough many arguments that the character of the problem does not qualify for the logarithmic chapter. Also, the referee of the book should have spotted this mistake, especially because of the last comment about more data.

\section{Making passwords}

In the textbook OFI [1] one can find easier examples in the combinatorics section. Some counting problems are dressed up to password creating questions. Here is a typical example:

Problem 4. Making password is a really important issue. Because of the so many passwords we can easily get in trouble, we can forget them. All of the computer passwords of Bence are composed of elements of the set $\{B, e, n, 1,7\}$. One document was benched with a 3-character password, but he forgot it. How many attempts will it take to reach the good one, if

a) he knows that he used three different character to create the password;

b) he does not know if he used one charachter only once?

Solution 4. a) There are 5 possibilities for the first character and 4 possibilities for the second one. It means that Bence can choose the first two characters in $5 \cdot 4=20$ different ways. Each of the 20 different options could be resumed in 3 different ways, which means that a total of $5 \cdot 4 \cdot 3=60$ passwords can be created. With 60 attempts at most Bence can find the password (making sure that the program allows so many attempts).

b) The first and the second characters can be of 5 types, as repetition may occur. The first two characters can therefore be chosen in $5 \cdot 5=25$ different ways. Each of the 25 different options could be resumed in 5 different ways, so Bence can create a total of $5 \cdot 5 \cdot 5=125$ passwords. With up to 125 attempts he can find the password. 
The following problem is posed after a series of easier counting problems in [1] in the same combinatorics section. It looks as typical as the previous one.

Problem 5. a) How many password can be made if you are only allowed to use the letters of the English alphabet and a password is 6 characters long.

At this point we do not answer the question, we simply copy here the next two problems, parts b and c:

Problem 6. [Problem 4 p. 14 [1]]

a) How many passwords can she choose from, if a password is 6 characters long and all letters has to be distinct.

b) How many passwords can she choose from, if a password is 6 characters long and repetition of characters is permitted.

c) How many passwords can she choose from, if she is allowed to use the small case and capital letters, as well.

We think that even a thorough reader gives the answer $26^{6}$ to part a), and $26 \cdot 25 \cdot 24 \cdot 23 \cdot 22 \cdot 21$ to part b). But then, after reading part c) becomes obvious that we are talking about a dressed up problem, and both answers are wrong. The correct answer to b) is $52^{6}$. The answer to part a) becomes more complicated, as we have to distinguish between letters and characters. Thus if you want to choose different letters, for the first character you can pick all 52 characters, and for the second one you have to exclude both the lower case and capital version of the first letter and have 50 choices. Thus the answer is $52 \cdot 50 \cdot 48 \cdot 46 \cdot 44 \cdot 42$. The end of the book contains a section: Answers to some problems. For this problem it says the following:

a) 165765600

b) 308915776

c) Multiply each answer by 128

Note that this answer to a) is equal to $26 \cdot 25 \cdot 24 \cdot 23 \cdot 22 \cdot 21$, to b) $26^{6}$. So their answer is incorrect. On the other hand, if you look at the real process of making a password, you are always allowed to use both lower case an capital letters. And in the past few years these home pages became very sensitive about safety and require a "safe" password. The criteria of these passwords are including most of the following requirements:

(1) the password should be at least 8 characters long 
(2) it should contain both lower case and capital letters

(3) it should contain numbers

(4) it should contain special characters

Hence the problem cannot be a modelling problem. It is a dressed up problem, but the dress-code went wrong, again. In addition, the textbook's answer to problem c) is incorrect even in their sense.

When posing or solving problem in combinatorics there is often a confusion about how to understand, how to interpret the actual problem. Is repetition allowed, do you put back the marbles after drawing, etc. are questions that arise during the implementation of counting problems. Are you allowed to touch a vertex twice, pick an edge multiply are questions when solving problems about graphs. In our case we might say that Problem 6 asks for only lower case letter passwords and it is clear from the context of part c). However, Problem 1 of the same chapter of [1] asks the following.

Problem 7 (Problem 1 p. 14 [1]). Hajni (nickname for the name Hajnalka) is making a password of character length 4 from the elements of the set $\{H, a, j, n, i, 1,9\}$. How many passwords can she choose from, if

(1) all characters have to be distinct.

(2) you can use a character as many times as you wish.

Thus in case solving the problems of the book in order, when arriving at Problem 4, pupils were aware of the distinction of capitals and lower case letters.

\section{Shortest paths}

One of the most popular textbook [12] for grade 9 students involves 6 chapters

(1) Combinatorics

(2) Number theory

(3) Coordinate-system, functions

(4) Equations and inequalities

(5) Triangles, rectangles, polygons, The circle and its parts

(6) Statistics

In the section Triangles, rectangles, polygons, the circle and its parts the following question is posed: 
Problem 8 (Problem 1 p. 100 [12]). Oil tank: There are four oil wells, so-called rockers, on an oil field. The oil is conducted from the rockers to the oil container by a transmission pipeline. Where should the container built in order to minimize the length of pipelines? (The rockers are located at the vertices of a convex quadrilateral: $A B C D$.)

The answer according to the teachers' handbook: The intersection of the diagonals. Sketch of the proof: let $P$ be an internal point of the quadrangle, which is not located on the diagonals. According to the triangle inequality $P A+P C>$ $A C, P B+P D>B D$, which means the total distance from the four vertices of the ABCD convex square to the point is longer than the distance of the two diagonals.

Both the answer and the reasoning are wrong. Where is the problem? Actually, there are several problems with the problem and the solution, as well.

1. At first, you do not have to be a careful reader or a good problem solver to see that once the pipeline configuration is fixed, the oil tank can be located anywhere along the pipeline: its location does not change the total length of the pipeline and gives a solution to the problem. It means, that the answer is not a point, the answer is a set of points. The real question is: "What is the pipe system, which meets the conditions of the problem?" Moreover, this also shows that the handbook's solution cannot be correct. Indeed, it proves that the solution is a single point and it cannot be true.

2. It is not even true that the sum of the diagonals is minimizing the length of the system. Here is a counter-example: Let $A B C D$ be a rectangle, where the angle of the diagonals is less then $60^{\circ}$. According to the textbook the solution would be the one on Figure 1.

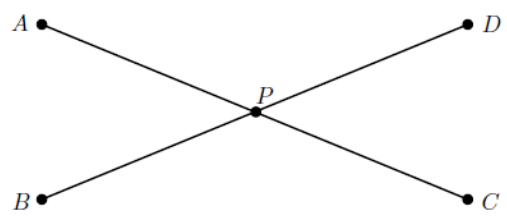

Figure 1. Solution proposed by the book 
However, since the angle of the diagonals is less then $60^{\circ}$ we have that $P A B \varangle<A P B \varangle$ so $P A>A B$ and that the path indicated on Figure 2. is shorter then the sum of the diagonals:

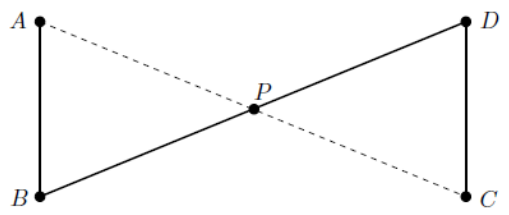

Figure 2. Improved solution

3. For 3 points (or oil wells) the pipeline must connect the three points with their Fermat point. Indeed, the Fermat point by definition is a point such that the total distance from the three vertices of the triangle is minimal. The angle of the lines connecting the Fermat point with the vertices of the triangle is 120 degrees. It follows that for the rectangle ABCD one can use the Fermat points of the triangles $\mathrm{ABP}$ and $\mathrm{PCD}$ to find a shorter pipesystem. Fourty years ago, in Hungary the Fermat point was included in the curriculum, now it is extracurricular.

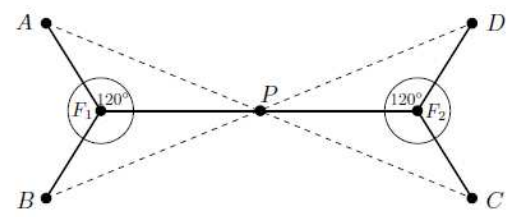

Figure 3. Optimal solution for a rectangle

In 1-3 we gradually improved the textbook's answer to the question, but what is the optimal solution to the problem in the generic situation? The solution is given by one of the Steiner trees of the points ABCD. For four points it is not dificult to construct the Steiner trees and there are at most three of them. The shortest one will be the solution to our problem. In case of several of points finding the shortest Steiner tree is algorithmically difficult, it is an NP-complete problem. 


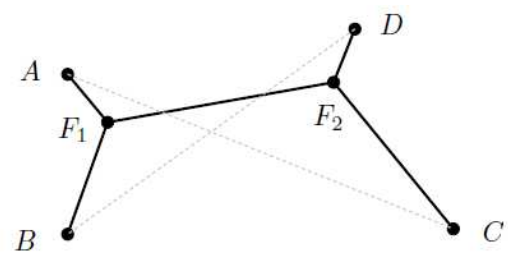

Figure 4. Optimal generic solution

As we have seen, the solution and the demonstration of the textbook is incorrect. The sample solution solves a different problem, not the one intended. Something went wrong in the modelling cycle: already the first point of the modelling process is erroneous (understanding the problem and constructing an individual situation modell), and also there are problems with the 6th step (validating the result with reference to the original situation and if the result is unsatisfactory, the process may start again with step 2). What could have happened? We found a possible explanation to these errors: the author wanted to ask a different question. Actually the textbook answers to a different problem: Which point minimizing the total distance from the four vertexes of the convex quadrilateral ABCD? Probably the author wanted to dress up the above pure geometrical problem but he was not careful enough by formulating it. In the intention to make the problem lively and realistic, and knowing that the shortest distance between two point is a line segment, the author changed the "segment" to "oil pipeline". However the pipelines are not necessarily "line segments" and from now on the task is to minimize the total distance os a system instead of finding a point which minimizes the total distance from the four vertexes of the convex quadrilateral ABCD. Consequently the dressing changes the context and changes the problem itself.

\section{The importance of responsibility}

Modelling and dressed-up problems play an inevitably unavoidable role in mathematics education. It is impossible to find a model from real life to every single topic and it is not a goal of math education. Dressed up problems can make semi-realistic flavour to intra-math problems making them more reachable for pupils. The teacher or book author has to be genuine and creative when 
dressing up math formulas or math ideas. In our paper we showed three different examples when the dress-code is flawed. In each three cases even the text of the problem suggests that the solution to the problems is not the ones the author believed. The danger in dressing up the problem is in Step 2 of the modelling cycle. This step is done towards when the pupil solves the problem and was created backwards when the dressing went on by the problem creator. Thus the validation part of modelling cannot be proceeded. In this case the students either try to figure out "what the teacher meant", or they solve a different problem. In two of our three cases this different problem is way ahead of pupils knowledge. The sample solution can be convincing enough to those who do not solve the problem themselves. And teachers can be mislead by the textbooks and pupils by the teachers.

\section{Acknowledgments}

The research was supported by the Hungarian Scientific Research Fund (OTKA) grant no. K109185 and by the Pázmány Eötvös Scientific Information Foundation.

The research was supported by the Mathlearnology Resrearch Group and the National Research, Development and Innovation Fund of Hungary, financed under the FK 124814 funding scheme.

\section{References}

[1] I. Barcza, I.Basa and M. Tamásné Kollár, Matematika tankönyv 11., OFI, Budapest, 2015.

[2] W. Blum and M. Niss, Applied mathematical problem solving, modelling, applications, and links to other subjects - State, trends, and issues in mathematics instruction. Educational Studies in Mathematics, 22, (1991), 37-68.

[3] J. Boaler, The role of contexts in the mathematics classroom: do they make mathematics more "real"?. For the Learning of Mathematics, 13(2), (1993) 12-17.

[4] J. Boaler, When do girls prefer football to fashion? An analysis of female underachievement in relation to 'realistic' mathematics contexts. British Educational Research Journal 20(5), (1994) 551-664.

[5] B. Cooper and M. Dunne, Assessing children's mathematical knowledge: social class, sex, and problem-solving. Open University Press, Buckingham, Philadelphia, 2000 . 
[6] M. Csordás, J. Kosztolányi, I. Kovács, K. Pintér, J. Urbán and I. Vincze, Sokszinü matematika 11., Mozaik Kiadó, Szeged, 2016.

[7] Cs. Csíkos, J. Szitányi and R. Kelemen(2011): The effects of using drawings in developing young children's mathematical word problem solving: A design experiment with third-grade Hungarian students. Educational Studies in Mathematics, 81, (2011) 47-65.

[8] S. Gerofsky, Genre analysis as a way of understanding pedagogy in mathematics education. For the Learning of Mathematics, 19(3), (1999) 36-46.

[9] G. A . Goldin and J. Kaput, A joint perspective on the idea of representation in learning and doing mathematics, in Theories of mathematical learning L. Steffe, P. Nesher, P. Cobb, G. Goldin, B. Greer (Eds.). Machwah (New Jersey), (1996) 397-430.

[10] B. Greer, The modeling perspective on world problems. Journal of Mathematical Behavior, 12, (1993) 239-250.

[11] M. Van den Heuvel-Panhuizen, The role of contexts in assessment problems in mathematics. For the Learning of Mathematics, 25(2), (2005) 2-9.

[12] J. Kornai, E. Kovács, É. Lövey, K. Pálovicsné Tusnády and M. Schubert, Matematika a középiskolák 9. évfolyama számára, Apáczai Kiadó, Celldömölk, 2009.

[13] De Lange, J. Assessment: No change without problems. Reform in school mathematics and authentic assessment (1995) 87-172.

[14] J. Lave, Word problems: A microcosm of theories of learning. in: Context and cognition: Ways of learning and knowing ), P. Light G. Butterworth (Eds.), Hemel:Harvester Hempstead, U.K. (1992) 74-92.

[15] Nemzeti Alaptanterv (2007) (National Core Curriculum), Available from: http://www.nefmi.gov.hu/kozoktatas/tantervek/nemzeti-alaptanterv-nat

[16] M. Niss, Applications and modeling in school mathematics-directions for future development, in Wirzup, I. and Streit, R. (eds), Developments in school mathematics education around the world 3, Chicago, National Council of Teachers of Mathematics, 1992.

[17] M. Niss, W. Blum and P. L. Galbraith, Introduction. In W. Blum, P. L. Galbraith, H.-W. Henn, M. Niss (Eds.), Modelling and Applications in Mathematics Education: the 14th ICMI Study. New York: Springer, (2007) (pp.13-2.)

[18] T. Palm, Word problems as simulations of real-world situations: A proposed famework. For the Learning of Mathematics, 26(1), (2006) 42-47.

[19] N. Presmeg, Visualisation in high school mathematics. For the learning of mathematics, 6, (1986) 42-46.

[20] K. Reusser, Problem solving beyond the logic of things: contextual effects on understanding and solving word problems. Instructional Science, 17, (1988) 309-338.

[21] S. Schukajlow, D. Leiss, R. Pekrun, W. Blum, M. Müller and R. Messner, Teaching methods for modelling problems and student's task-specific enjoyment, value, interest and self-efficacy expectations. Educational Studies in Mathematics, 79(2), (2012) 215-237. 
[22] D. Stocker, Re-Thinking Real-World Mathematics. For the Learning of Mathematics, 26(2), (2006) 29-30.

[23] L. Verschaffel, E. De Corte and S. Lasure, Realistic considerations in mathematical modeling of school arithmetic word problems. Learning and Instruction, 4(4), (1994) 273-294.

[24] T. Wedege, To know - or not to know - mathematics, that is a question of context, Educational Studies in Mathematics, 39, (1999) 205-227.

[25] http://drogfokuszpont.hu/eves-jelentesek/

[26] http://www.emcdda.europa.eu/countries/hungary\#drd

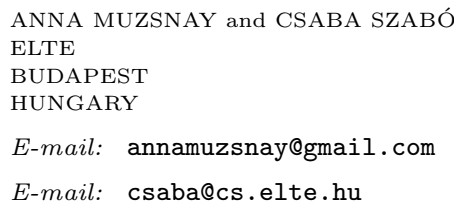

